\title{
Performance studies under high irradiation of resistive bulk-Micromegas chambers at the CERN Gamma Irradiation Facility
}

\section{B. Alvarez Gonzalez*, CERN, E-mail: balvarezdcern.ch}

J. Bortfeldt, CERN, E-mail: jonathan.bortfeldtecern.ch

E. Farina, CERN and Universita e INFN, Pavia, E-mail:

edearde.maria.farinadcern.ch

P. lengo, CERN, E-mail: Paolo.Iengodcern.ch

L. Longo, Universita del Salento, E-mail: Luigi.longodcern.ch

O. Sidiropoulou, CERN and Julius-Maximilians-Universität Würzburg, E-mail:

ourania.sidiropouloudcern.ch

J. Wotschack, CERN, E-mail: Joerg.Wotschackdcern.ch

Radiation studies on several resistive bulk-Micromegas chambers produced at CERN will be viewed in this document. Two resistive bulk-Micromegas chambers have been installed at the CERN Gamma Irradiation Facility (GIF++) exposed to an intense gamma irradiation with the aim of evaluating the detector behaviour under high irradiation and carrying out a long-term ageing study.

The chambers under study have an active area of $10 \times 10 \mathrm{~cm}^{2}$, a strip pitch of $400 \mu \mathrm{m}$, an amplification gap of $128 \mu \mathrm{m}$, and a drift gap of $5 \mathrm{~mm}$.

The results on the detector performance as a function of the photon flux up to $44 \mathrm{MHz} / \mathrm{cm}^{2}$ will be shown as well as the ageing properties as function of the integrated charge and the current intensity and its stability with time. In addition, the results of the efficiency measurements before, during, and after the irradiation will also be presented as a function of the amplification voltage at which the chambers are operated.

38th International Conference on High Energy Physics

3-10 August 2016

Chicago, USA

${ }^{*}$ Speaker. 


\section{Introduction}

Two resistive bulk-Micromegas, with active area of $10 \times 10 \mathrm{~cm}^{2}$, produced at the CERN PCB workshop have been installed at the CERN Gamma Irradiation Facility (GIF++) with the aim of studying their behaviour under high irradiation and carrying out a long-term ageing study. The Micromegas technology has been considered for future upgrade projects of large scale experiments like for the ATLAS [U] New Small Wheel (NSW) upgrade project of the Muon Spectrometer [व].

The detectors are exposed to an intense gamma $(\gamma)$ irradiation of flux up to $44 \mathrm{MHz} / \mathrm{cm}^{2}$ provided by the $13.9 \mathrm{TBq}{ }^{137} \mathrm{Cs}$ source of GIF++. This corresponds to 10 times more than the highest expected counting rate at the High-Luminosity Large Hadron Collider (HL-LHC) in the NSW region. The resistive bulk-Micromegas chambers have been exposed during several months at GIF++ and will be kept in the irradiation position in order to accumulate an integrated charge up to $\sim 0.2 \mathrm{C} / \mathrm{cm}^{2}$ which corresponds to the highest expected flux in the NSW for about 10 years of HL-LHC operation.

An overview of the ongoing tests at GIF++ in terms of hit rate, integrated charge and spatial resolution of the Micromegas detectors is given in this document.

\section{Gamma Irradiation Facility at CERN}

The Gamma Irradiation Facility [3], 团] is located in the North Area of the CERN Super Proton Synchrotron (SPS) and has been operational since March 2015. It is a unique place where high energy charged particle beams (mainly muons) are combined with a flux of photons from a $13.9 \mathrm{TBq}$ ${ }^{137}$ Cs source, about $50 \%$ of the photon current comes from photons with energy $662 \mathrm{keV}$. A filter system permits attenuating the photon rate in several steps to reach attenuation factors of several orders of magnitude $\left(\sim 10^{4}-10^{5}\right)$. The high source activity produces a gamma field intense enough to accumulate doses equivalent to HL-LHC experimental conditions in a reasonable time. The Micromegas detectors are situated in the upstream area at $1 \mathrm{~m}$ from the source exposed to a $\gamma$ flux up to $44 \mathrm{MHz} / \mathrm{cm}^{2}$.

\section{Description of the Micromegas used in GIF++}

Micromegas are gaseous particle detectors detecting particles by amplifying the charges that have been created by ionisation in the gas volume. This gas volume is divided in two regions by a metallic micro-mesh allowing for a high gain of $10^{4}$ and a fast signal collection time of $100 \mathrm{~ns}$, Fig. W. Two resistive bulk-Micromegas detectors [G] are used in GIF++ for the studies described in this document. These chambers, called T5 and T8 (shown in Fig. (2), have an active area of 10x10 $\mathrm{cm}^{2}$, a single readout plane with strip pitch $400 \mu \mathrm{m}$ and strip width $300 \mu \mathrm{m}$. The readout strips are covered with a $50 \mu \mathrm{m}$ thick insulator carrying high resistivity $(\sim 1 \mathrm{M} \Omega / \mathrm{sq})$ carbon strips for spark protection. The mesh consists of $18 \mu \mathrm{m}$ diameter wires with $64 \mu \mathrm{m}$ pitch. The amplification gap is $128 \mu \mathrm{m}$, and the drift gap is fixed at $5 \mathrm{~mm}$ with a drift field of $600 \mathrm{~V} / \mathrm{cm}$.

\subsection{Data-taking and working conditions}

APV-25 front-end ASICs [ [ $]$ ] and the RD51 Scalable Readout System (SRS)[8] have been used for the data-taking. The measurements were performed varying the amplification voltage 


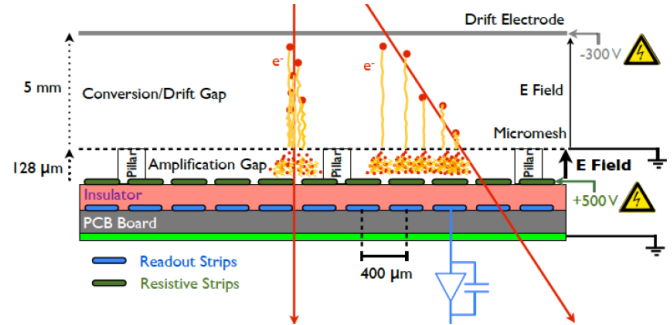

Figure 1: Micromegas detector schema. HV numbers and dimensions correspond to the typical working conditions.

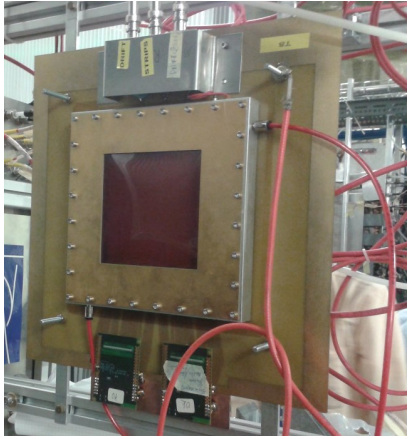

Figure 2: T8 chamber installed in GIF++.

and the attenuation filters. The following attenuation factors $[1,1.5,2.2,4.6,6.9,10,22,33,46$, $69,10^{2}$ ] have been used. The amplification voltages used go from 420 to $540 \mathrm{~V}$ in $10 \mathrm{~V}$ steps, that corresponds to an amplification field of $33-42 \mathrm{kV} / \mathrm{cm}$. The working conditions during the measurements were as follows: $\mathrm{Ar} / \mathrm{CO}_{2} 93 \% / 7 \%$, gas flow $5 \mathrm{l} / \mathrm{h}$, and operating gain of $\sim 2 \times 10^{4}$ at $540 \mathrm{~V}$.

\section{Hit Rate and Detector Sensitivity}

Data have been collected with several attenuation filters to estimate the hit rate. The hit rate is defined as the average number of converted photons counted in a time window of $625 \mathrm{~ns}$ divided by the time window length and the active area. Figure 3 shows the measured hit rate $\left(\mathrm{Hz} / \mathrm{cm}^{2}\right)$ as a function of the amplification voltage for the different attenuation factors (see the legend of the figure). From the measured rate in the plateau region (at $520 \mathrm{~V}$ ) and the simulated flux the detector sensitivity to $\gamma$ 's is extracted to be approximately $3.5 \times 10^{-3}$.

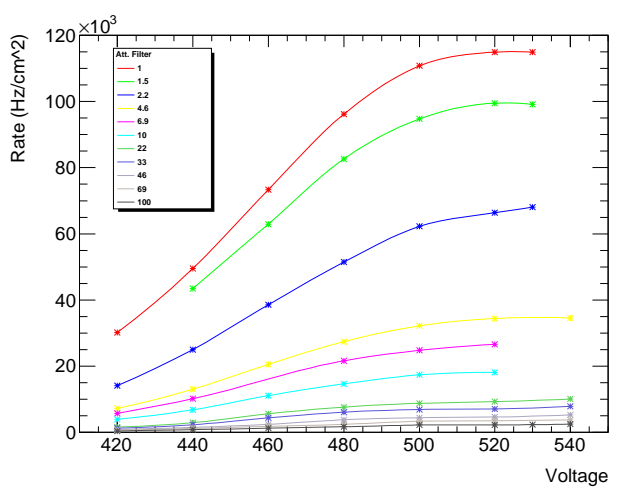

Figure 3: The measured particle rate as a function of the amplification voltage for 11 different $\gamma$ rates.

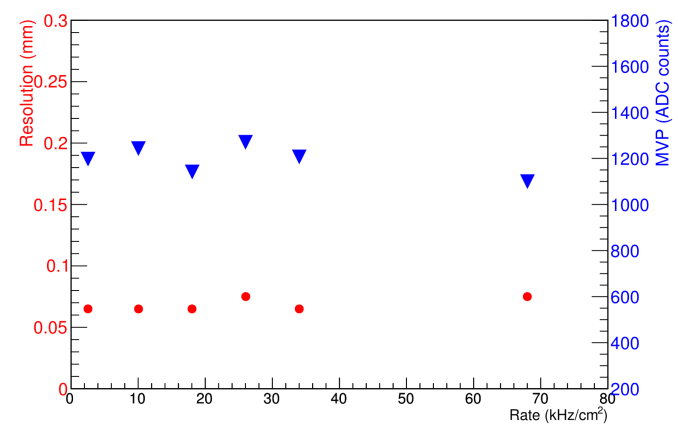

Figure 4: Resolution $[\mathrm{mm}]$ and the most probable value (ADC counts) of the clusters as a function of the measured rate $\left[\mathrm{kHz} / \mathrm{cm}^{2}\right]$.

\section{Tracking with muon beam}

GIF++ provides for the possibility of combining the source with a high energy muon beam 
which has been used for some resolution studies presented in this section. During the muon beam data-taking, in addition to the T5 and T8 detectors two other chambers are installed as reference chambers in order to define muon tracks. A tracking algorithm using the Hough transform [Q] requires at least one cluster in all the layers to distinguish muons from photons. Then the distance between the cluster positions on T5 and T8 is calculated. The cluster position difference is fitted with a Gaussian function. Assuming that both chambers have the same resolution, the final resolution is taken as the sigma of the Gaussian fit divided by $\sqrt{2}$ leading to $\sim 65-70 \mu \mathrm{m}$ resolution.

The analysis has been repeated for all attenuation points and results in the resolution as a function of the measured rate as shown in Fig. $⿴$. The resolution and the most probable value of the clusters are flat and stable up to $68 \mathrm{kHz} / \mathrm{cm}^{2}$ (4 times more than the expected rate during the HL-LHC in the NSW region) which means that detectors operate without any performance degradation or gain reduction. It also means that the algorithm used to select the muon candidates works successfully and provides for the same resolution under high radiation conditions. No data were collected with muon beams above the measured hit rate of $68 \mathrm{kHz} / \mathrm{cm}^{2}$ corresponding to an attenuation factor 2.2.

\section{Summary and Plans}

Aging studies are mandatory to asses the capability of the detectors to handle the rate and level of radiation at the HL-LHC. The two resistive bulk-Micromegas chambers have been exposed to an intense gamma radiation in $\mathrm{GIF}++$ for several months. The acummulated integrated charge over more than 10 months of irradiation at GIF++ is about $130 \mathrm{mC} / \mathrm{cm}^{2}$. No degradation of the detector performance has been observed. The goal is to accumulate more than $0.2 \mathrm{C} / \mathrm{cm}^{2}$, the equivalent charge expected in 10 years of HL-LHC operation in the NSW region. The detector ageing evolution will be checked in terms of dark and amplification currents, efficiency and noise stability as a function of the integrated charge.

\section{References}

[1] ATLAS Collaboration, JINST 3 S08003 (2008).

[2] ATLAS collaboration, ATLAS-TDR-20-2013.

[3] M. R. Jäkel et al., CERN GIF++, PoS (TIPP2014) 102

[4] http://ph-dep-dt.web.cern.ch/irradiation-facilities/gif

[5] D. Pfeiffer et al., https://arxiv.org/abs/1611.00299

[6] T. Alexopoulos et al., Nucl. Instr. Meth. Phys. Res. A 640 (2011) 110-118

[7] M. Raymond et al., IEEE Nucl. Sci. Symp. Conf. Rec. 2 (2000), 9/113

[8] S. Martoiu et al., JINST 8 C03015 (2013)

[9] P. V. C. Hough, Proc. Int. Conf. on High Energy Accelerators and Instrumentation, 1959 